\title{
A Novel Intrusion Detection System in WSN using Hybrid Neuro-Fuzzy Filter with Ant Colony Algorithm
}

\author{
'Sarah Salaheldin Lutfi, \& ${ }^{2}$ Mahmoud Lutfi Ahmed \\ ${ }^{1}$ Aysik Consulting Services, Evans GA, USA. \\ ${ }^{2}$ Georgia Southern University, Statesboro, GA 30458, USA \\ *Corresponding Author: sarahlutfi7@gmail.com
}

Received: 02.01 .2020 , Revised: 05.02 .2020 , Accepted: 15.03 .2020 , Published: 30.03 .2020

DOI:

10.53409/mnaa.jcsit1101

Abstract: With the wide application of wireless sensor networks in military and environmental monitoring, security issues have become increasingly prominent. Data exchanged over wireless sensor networks is vulnerable to malicious attacks due to the lack of physical defense equipment. Therefore, corresponding schemes of intrusion detection are urgently needed to defend against such attacks. A new method of intrusion detection using Hybrid Neuro-Fuzzy Filter with Ant Colony Algorithm (HNF-ACA) is proposed in this study, which has been able to map the network status directly into the sensor monitoring data received by base station, accordingly that base station can sense the abnormal changes in network. The hybridized Sugeno-Mamdani based fuzzy interference system is implemented in both the NF filters to obtain more efficient noise removal system. The Modified Mutation Based Ant Colony Algorithm technique improves the accuracy of determining the membership values of input trust values of each node in fuzzy filters. To end, the proposed method was tested on the WSN simulation and the results showed that the intrusion detection method in this work can effectively recognise whether the abnormal data came from a network attack or just a noise than the existing methods.

Keywords: Hybrid Neuro-Fuzzy Filter, Ant Colony Algorithm, fuzzy filters, wireless sensor networks, security issues.

\section{INTRODUCTION}

ttacks in Wireless Sensor Networks (WSNs) aim in limiting or even eliminating the ability of the network to perform its expected function. WSNs are networks with limited resources and often deployed in uncontrollable environments that an intruder can easily access. WSN attacks target specific network layer's vulnerabilities but normally affect other layers as well. Local sensor activity at multiple sensor network layers should be monitored and evaluated to detect possible malicious intervention. Intrusion detection is the method of analyzing and checking for signs of potential accidents in a computer device or network and also of unauthorized access. Intrusion detection This normally occurs by gathering data from a number of devices and network sources automatically, again and reviewing the data for safety issues.

According to [1], the annual cost from cyber-crimes to the global economy is estimated to be more than $\$ 400$ billion in 2014. This statistic stresses the importance of developing proper solutions to ensure the safety of information systems. In order to protect information systems against intruders, different preventive solutions have been deployed by organizations. Intrusion detection system, a type of security management system used to gather and analyze information on a computer network or on a website to help build a different attack safeguard mechanism [2]. Also it is recording massive research works on its system using data mining which have been flooded by many researchers recently as it have the potency of classifying and detecting anomalies within a network.

In many real-time applications, there is a need to secure the communication and perform an effective data transmission. Due to attacks and some hackers, various security issues may occur. A lot of researchers were focussed but since the tools such as anti-virus programs and firewalls are not sufficient to provide constant and reliable sources of information. There is a 
need to test the IDS system by upgrading the features of the system and provide integrity.

This work focuses on Hybrid Neuro-Fuzzy Filter with Ant Colony Algorithm (HNF-ACA) helps to predict the malicious node with data authentication. This approach is distinct from the standard algorithms for intrusion detection that treat network traffic condition directly. The innovation is that the network status is mapped to a base station's measurements to reflect changes in network status.

The rest of the paper is structured as follows: In section 2 the related work of IDS in WSN is discussed. The proposed IDS detection mechanism using Hybrid Neuro-Fuzzy Filter with Ant Colony Algorithm (HNF-ACA) is described in section 3 . The experimental results and discussion is discussed in section 4 . The conclusion and future work is given in section 5 .

\section{RELATED WORK}

In literature, there are a few works that aim to combine between anomaly-based approach and hybrid model to benefit from the advantages of both detection techniques. In [3] proposed a mechanism of Intrusion Detection System (IDS) created in a Cluster-based Wireless Sensor Network (CWSN). The method of feature selection is one of the important factors, which affects the performance of IDS. In the field of wireless sensor networks an IDS architecture inspired by the Human Immune System is proposed[4]. However, this intrusion detection systems are too resource-demanding.

A hybrid clustering method is introduced in [5], namely a density-based fuzzy imperialist competitive clustering algorithm (D-FICCA). However, studying a game-based evolutionary algorithm is considered extremely significant. In [6] proposed a lightweight, energy-efficient system, which makes use of mobile agents to detect intrusions based on the energy consumption of the sensor nodes as a metric. A linear regression model is applied to predict the energy consumption. In [7], a game theoretic method is introduced, namely cooperative Game-based Fuzzy Q-learning (G-FQL). G-FQL adopts a combination of both the game theoretic approach and the fuzzy Q-learning algorithm in WSNs. The energy consumption for intrusion detection and prevention should be focused.
In [8] proposed an appropriate probabilistic model which provides the coverage and connectivity in k-sensing detection of a wireless sensor network. However, when random deployment is required, determining the deployment quality becomes challenging. In [9] proposed a Trust Based Adaptive Acknowledgment (TRAACK) IntrusionDetection System for WSN based on number of active successful deliveries, and Kalman filter used to predict node trust. Based on hybrid models this work is proposing an efficient hybrid system for sensor network. The goal in this research is to study and implement a new model of intrusion detection that combines the advantages of hybrid model in cluster wireless sensor environment, and surpassing other models proposed in the literature.

\section{PROPOSED METHODOLOGY}

Initially, the fuzzy based Hybrid NeuroFuzzy Filter with Ant Colony Algorithm (HNFACA) is considered here to evaluate the detection of malicious nodes presented in the wireless sensor nodes. Generally, the malicious node is stated as the node that does not follow the exact behavior, it means there is any malicious attack such as modifying the message, in some terms the packet may be dropped likewise it goes on. Initially, there is a need for establishing the cluster based routing along with the reputation of a node. Then it is necessary to identify the malicious nodes, these parameters are based on the trust recommendation value. If the process is selected then the routing process is made by maintaining the process of each route. It is necessary to update the neighbor node information to source and destination nodes. Finally, for encryption, the RSA (RivestShamir-Adleman) scheme is used to provide the authentication to all nodes based on public and private keys. At last the HNF-ACA is used for classifying the normal and malicious nodes for efficient data transmission in WSN. The architecture diagram of HNF-ACA based IDS is illustrated in Figure 1. 


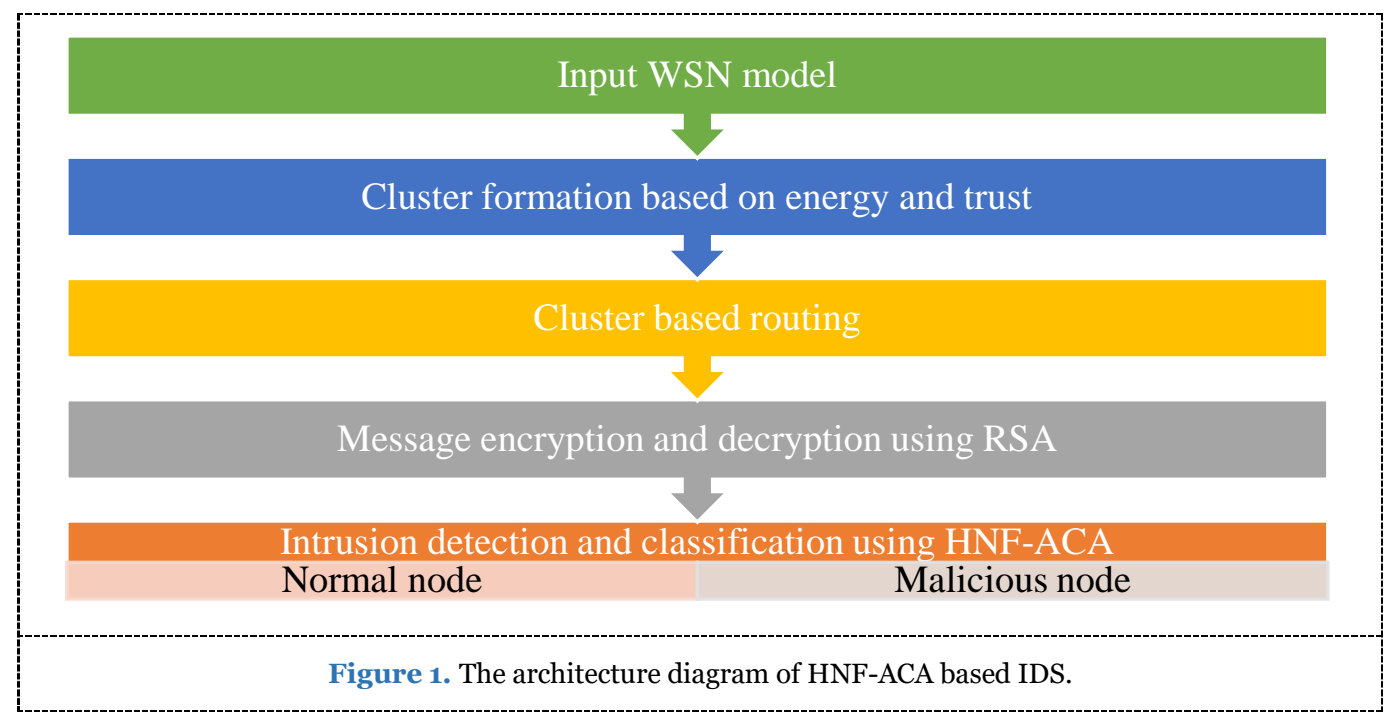

\subsection{Cluster Based Routing}

The cluster based routing is one of the efficient routing methods, here the nodes are selected for processing as well as sending the data, it is done with the help of high energies. In this work, the Cluster is framed by the nodes that are based on the parameters of residual energy and the trust vector value of reliable nodes. The trust is evaluated based on the successful delivered of packets among source and destination. The routing table of each node consists of watch dog mechanism to monitor the neighbor nodes data and it is necessary to inform the activity. Normally, the routing table is made up of packet id, neighbor coverage range, packet information, cluster member id, link quality, a node to node connectivity etc. The residual energy Res of each node $i$ at time $t$ is calculated as in equation (2), for each and every node to improve the network lifetime [10].

$$
\operatorname{Res}_{i}(t)=\frac{\left[\text { Initial }-E_{i}(t)\right]}{r e g}
$$

where Initial is the initial energy, $E_{i}(t)$ is the residual energy and reg is the current region of cluster formation. With respect to the high and medium trust vector value the process is determined, the processes of nodes are less energy consumption of the node are considered as a cluster head or cluster members. The trust value Trust $_{i}$ is calculated as in equation (2),

$$
\text { Trust }_{i}=\frac{\text { Deliv }_{i}-\text { packet }_{i}}{\text { recpacket }_{i}}
$$

where, Deliv $_{i}$ is described as the delivered packets from $D_{i}$, packet ${ }_{i}$ describes the packets sent from $D_{i}$ to $S_{i}$, recpacket rescribes $_{i}$ is descris received packets sent from $S_{i}$ to $D_{i}, D_{i}$ is the destination node and $S_{i}$ is the source node.

The trust value evaluation is made here to check highest trust value node and find the trusted authenticator. This authenticator indicated as Cluster Head $(\mathrm{CH})$ and other related trust value nodes are selected as cluster member nodes. In case, the selected node is already a member of another cluster region, then a node of high trust value is selected. Based on these $\mathrm{CH}$ and members, the cluster region is formed.

Authentication of Message by RSA: The message authentication is processed by RSA algorithm. One of the most popular methods for public key encryption is Ron Rivest, Adi Shamir and Leonard Adleman (RSA) and it is one of the secure publickey encryption methods [12].

\subsection{Classification Using HNF-MMACA}

To represent the original node representation the authenticate node is used and for misbehavior representation, the malicious node is considered. Based on these two factors the training class is defined. Consider a proposed HNF-MMACA system that has $N$ number of nodes in the input samples. The two classes are considered here in the training example data, i.e., the process has two labels, as well as the proposed system use $K=2$ like class groups of hidden nodes, and these nodes represents a Gaussian function midpoint with a correlated label. The process is a class grouped; each Gaussian has a different centre but the same label. 
Proposed Hybrid Neuro - Fuzzy: Structure of the Hybrid Neuro - Fuzzy, the fuzzy system is classified into Mamdani and Sugeno, which is associated with fuzzy rules. The structures of the two hybrid NF are identical to each other. Each filter is a combination of first order Sugeno and Mamdani fuzzy interference system. Therefore, the general form of the output function is given by equation (3),

$$
Y=\sum_{k=1}^{K} \frac{a_{k} v_{k}}{\sum_{k=1}^{K} a_{k}} \mathrm{~b}_{\mathrm{k}}(\mathrm{X})
$$

Where $a_{k}$ is the degree, the input x matches the rule computed, $b_{k}$ for the Sugeno model and volume of the output fuzzy set is represented as $v_{k}$ and the centroid of the output fuzzy set is denoted as $\mathrm{N}_{\mathrm{k}}$. The outcome of both the hybridized NF filter is computed by equation (10), represents the generalized fuzzy interference model which is hybridized the both Sugeno model and Mamdani model. Therefore, the hybridized model is defined as in equation (4)

$$
\begin{array}{r}
\text { Rule } k \text { : if } x_{1} \text { is } M_{1}^{k} \text {; } x_{2} \text { is } M_{2}^{k} ; \\
x_{3} \text { is } M_{3}^{k} \text { and } x_{4} \text { is } M_{4}^{k} \\
Y \text { is } N_{k}\left(v_{k}, b_{k}(X)\right)
\end{array}
$$

Where the $\mathrm{N}_{\mathrm{k}}$ for Mamdani model, or $\mathrm{b}_{\mathrm{k}}$ for the Sugeno model. The $\mathrm{M}_{\mathrm{i}} \mathrm{k}$ is represented [13] the membership function of the ith input of the kth rule. The essential features are preserved the interpretable capability of the Mamdani model and also enhance the accuracy of the Sugeno model as in equation (5). The hybrid NF outcome fetched into the post processor $Y_{\text {post }}$ is truncated to the 8 bit integer values.

The final outcome of the proposed filter $Y_{\text {Final }}$ is determined by the average output of the two hybrid NF filters. The function round (), rounds the element $x$ to the nearest integer.

$Y_{\text {Final }}=$ round $\left(\frac{1}{2} \sum_{\text {post }=1}^{2} Y_{\text {post }}\right)$

The outcome is fetched into the post processor post to produce the final output of the filter. The internal parameters of the hybridized NF filters are optimized using hybrid learning rule to reduce the error. The antecedent and the consequent parameters are optimized using gradient descendent and least mean squares algorithm respectively.
MACA based optimization of Membership values: The membership values are assigned for further optimizing the input to improve the accuracy of the system as it decides the efficiency of IDS [14].

Table 1. Algorithm for optimal membership value Selection using ACA for HNF based IDS

Input: Initial Membership values for nodes, $\mathrm{N}$ that represents the number of features (i.e nodes parameters)

Output: Optimized Membership values

Formulate the optimization problem as a graph that is fully connected.

Assign, initial values of membership to the nodes in the network model.

Let number of membership values be $m$;

While best membership value is not optimum do

Assign the parameter Residual energy function is minimum

Spread the membership values on the construction graph.

Update the visited membership values with their error functions.

While optimal membership value is not met do

\section{Initialize ACA}

For each ant do

Choose next node by applying state transition rule

Apply step by step pheromone update

End for

Repeat step 10 to 13 until met stopping criterion

Update best solution

Apply offline pheromone update

Check stopping condition not satisfied do

Position each ant in a starting node

For

. Check the Residual energy function of the value

End for

End while

Select best membership value in the values $m$ as $\mathrm{M}_{\text {best }}$; 


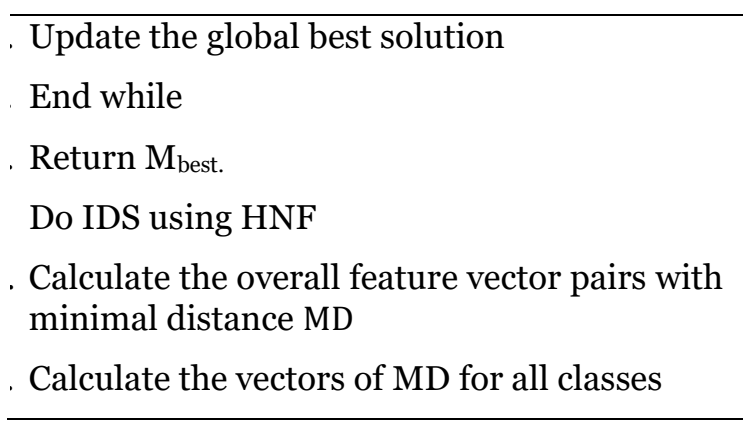

\begin{tabular}{l}
\hline Input can be classified based on the \\
conditions using equation (4) \\
If the classification is made for all inputs, then \\
the function is terminated, \\
Else forward it to the Step 31 process. \\
Produce the classification results of normal \\
and malicious node
\end{tabular}

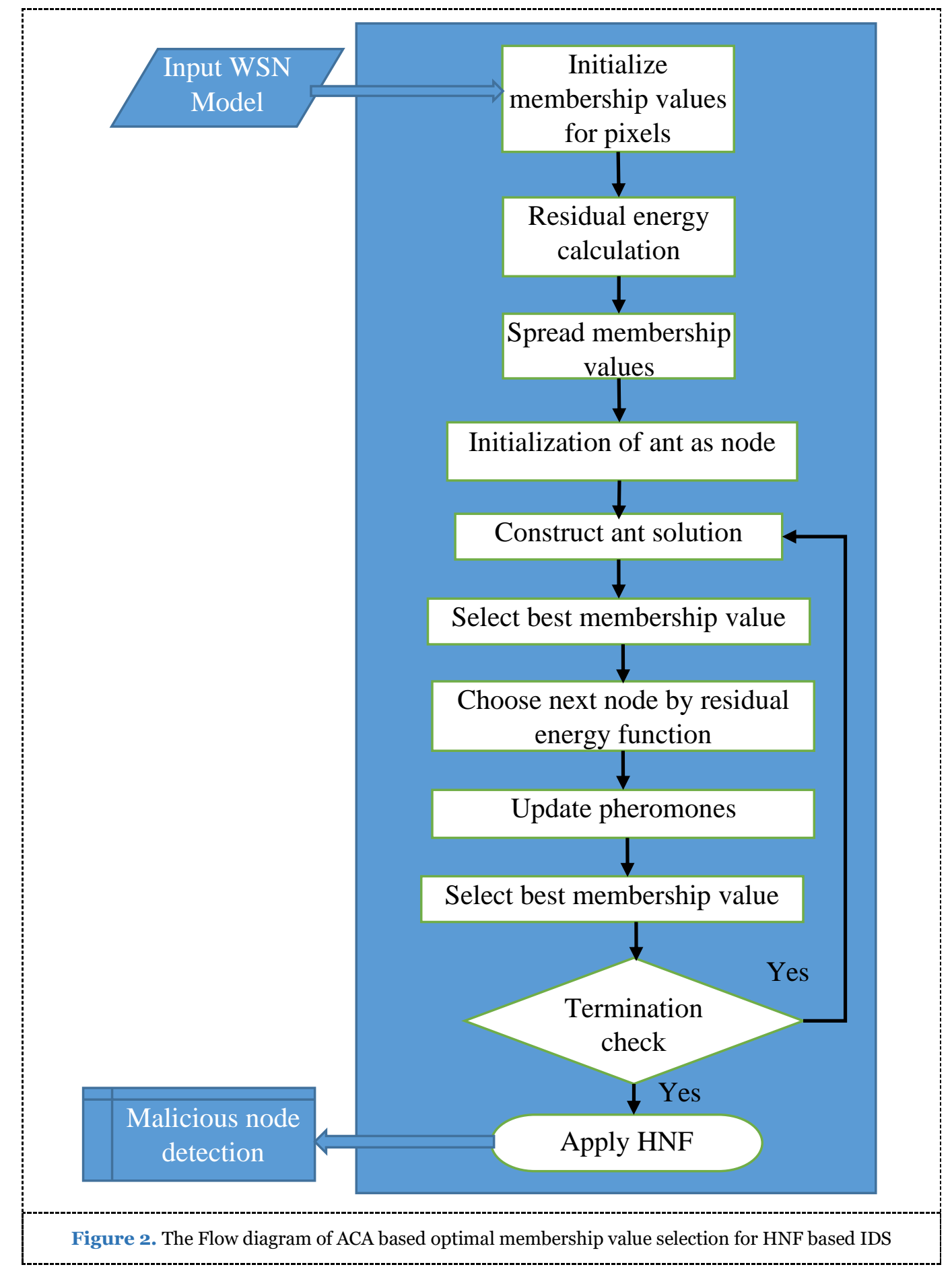




\section{EXPERIMENTAL RESULTS AND DISCUSSION}

In this section, the proposed HNF-ACA performance is carried out with the traditional methods such as D-FICCA [5], G-FQL [7] and TRAACK [9] in presence of malicious node environment in terms of Detection Rate Comparison, communication overhead, End to End delay and network lifetime. The proposed IDS are simulated with Network Simulator tool (NS 2.34). The simulation settings and parameters of the proposed scheme are given in Table 2 [15].

Table 2. Simulation Settings

\begin{tabular}{|c|c|}
\hline Number of nodes & 101 \\
\hline Area size & $1000 \times 1000$ \\
\hline Mac & 802.11 \\
\hline Radio range & $250 \mathrm{~m}$ \\
\hline Simulation time & $100 \mathrm{sec}$ \\
\hline Packet size & 8 obytes \\
\hline Mobility model & Random way point \\
\hline Protocol & LEACH \\
\hline
\end{tabular}

\subsection{Detetection Rate Comparison}

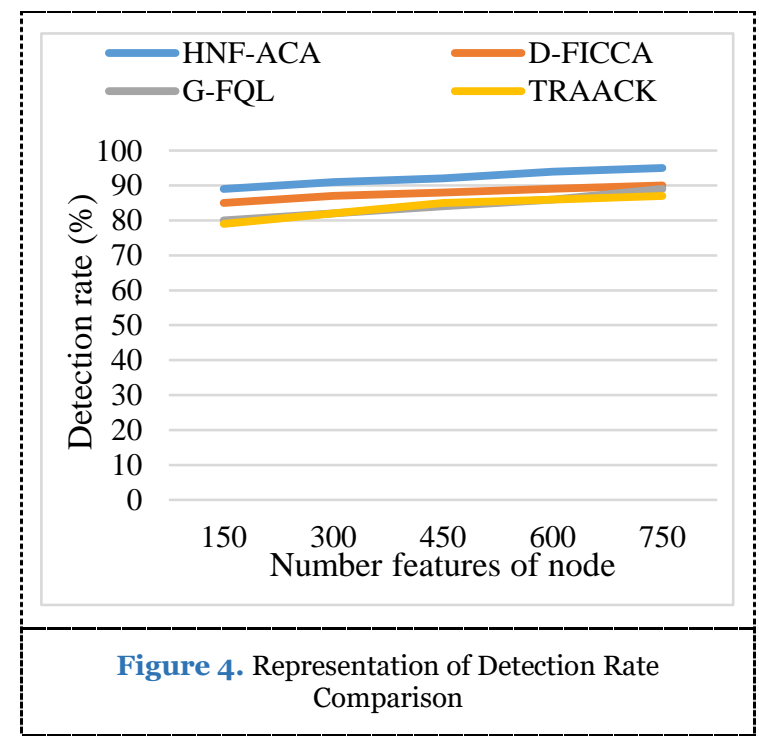

From the figure 4, when increasing the number of features the detection rate is increased twice that of the initial conditions. It is concluded that the detection rate of the proposed HNF-ACA method has improved with rate of $95 \%$. In exiting methods such as D-FICCA, G-FQL and TRAACK attains low rate of $90 \%, 89 \%$ and $87 \%$ respectively. The numerical results of detection rate comparison is shown in Table 3.
Table 3. The numerical results of detection rate comparison

\begin{tabular}{ccccc}
\hline $\begin{array}{c}\text { No.of } \\
\text { features }\end{array}$ & $\begin{array}{c}\text { HNF- } \\
\text { ACA }\end{array}$ & $\begin{array}{c}\text { D- } \\
\text { FICCA }\end{array}$ & $\begin{array}{c}\text { G- } \\
\text { FQL }\end{array}$ & TRAACK \\
\hline 150 & 89 & 85 & 80 & 79 \\
\hline 300 & 91 & 87 & 82 & 82 \\
\hline 450 & 92 & 88 & 84 & 85 \\
\hline 600 & 94 & 89 & 86 & 86 \\
\hline 750 & 95 & 90 & 89 & 87 \\
\hline
\end{tabular}

\subsection{Communication Overhead Comparison}

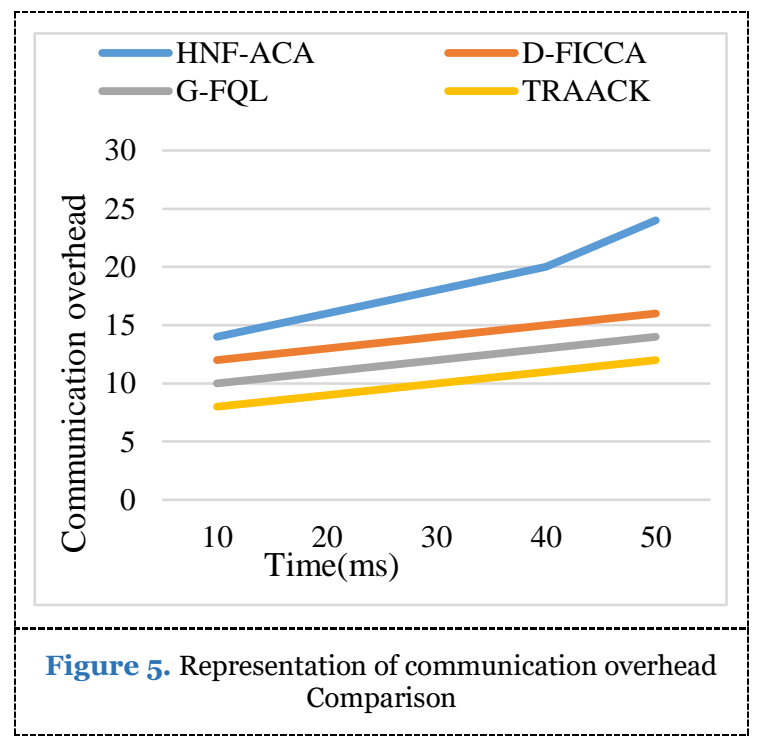

Communication overhead is a process of integrating an additional or indirect measure of resource that includes time, memory, bandwidth and a few other resources that are necessary to obtain the objective. From the figure 4, when increasing the time the communication overhead rate is increased. The communication overhead rate of the proposed HNF-ACA method has improved with rate of 24. In exiting methods such as DFICCA, G-FQL and TRAACK attains low rate of 16 , 14 and 12 respectively. The numerical results of communication overhead Comparison is shown in Table 4 .

Table 4. The numerical results of communication overhead Comparison

\begin{tabular}{ccccc}
\hline $\begin{array}{c}\text { Time } \\
(\mathbf{m s})\end{array}$ & $\begin{array}{c}\text { HNF- } \\
\text { ACA }\end{array}$ & $\begin{array}{c}\text { D- } \\
\text { FICCA }\end{array}$ & $\begin{array}{c}\text { G- } \\
\text { FQL }\end{array}$ & TRAACK \\
\hline 10 & 14 & 12 & 10 & 8 \\
\hline 20 & 16 & 13 & 11 & 9 \\
\hline 30 & 18 & 14 & 12 & 10 \\
\hline 40 & 20 & 15 & 13 & 11 \\
\hline 50 & 24 & 16 & 14 & 12 \\
\hline
\end{tabular}

6 | P a g e 


\subsection{End to end delay Comparison}

Figure 6 shows the result of an end to end delay by varying nodes mobility. The obtained delay graph shows that the delay taken by data packets to reach the destination is less for proposed HNF-ACA methodology with $0.2 \mathrm{~ms}$ when comparing with the traditional D-FICCA, G-FQL and TRAACK attains low rate of $0.24 \mathrm{~ms}, 0.25 \mathrm{~ms}$ and $0.31 \mathrm{~ms}$ respectively. The graph proves that the end to end delay is reducing if the mobility of the nodes gets increased.

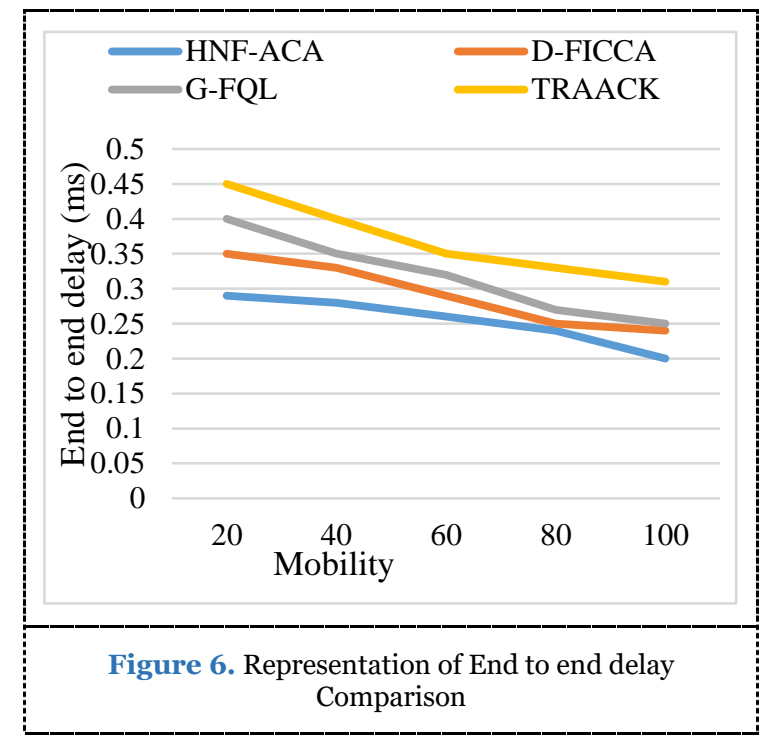

Hence, it is summarized that, if nodes get increased then the end to end delay decreased, it is stated as the transmission is effective. The numerical results of End to end delay Comparison is shown in Table 5 .

Table 5. The numerical results of End to end delay Comparison

\begin{tabular}{ccccc}
\hline Mobility & $\begin{array}{c}\text { HNF- } \\
\text { ACA }\end{array}$ & $\begin{array}{c}\text { D- } \\
\text { FICCA }\end{array}$ & $\begin{array}{c}\text { G- } \\
\text { FQL }\end{array}$ & TRAACK \\
\hline 20 & 0.29 & 0.35 & 0.4 & 0.45 \\
\hline 40 & 0.28 & 0.33 & 0.35 & 0.4 \\
\hline 60 & 0.26 & 0.29 & 0.32 & 0.35 \\
\hline 80 & 0.24 & 0.25 & 0.27 & 0.33 \\
\hline 100 & 0.2 & 0.24 & 0.25 & 0.31 \\
\hline
\end{tabular}

\subsection{Network Lifetime Comparison}

Figure 6 shows the result of Network lifetime by varying nodes time. The obtained delay graph shows that the delay taken by data packets to reach the destination is less for proposed HNF-ACA methodology with $1.21 \mathrm{~s}$ when comparing with the traditional D-FICCA, G-FQL and TRAACK attains low rate of $1 \mathrm{~s}, 0.9 \mathrm{~s}$ and $0.8 \mathrm{~s}$ respectively.

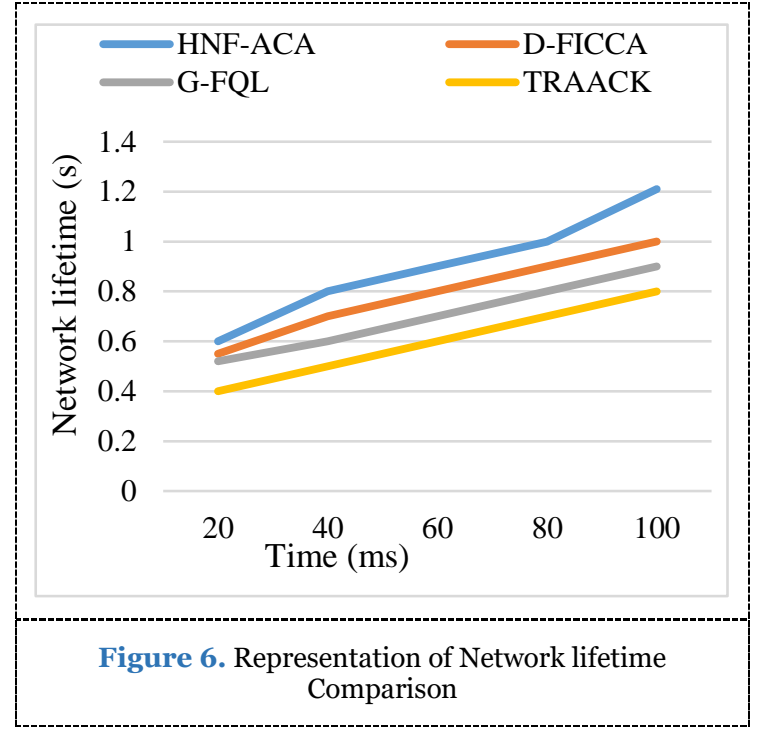

Hence, it is summarized that, the proposed HNFACA is highly recommended for IDS in WSN. The numerical results of Network lifetime Comparison is shown in Table 7.

Table 7. The numerical results of Network lifetime Comparison

\begin{tabular}{ccccc}
\hline $\begin{array}{c}\text { Time } \\
(\mathbf{m s})\end{array}$ & $\begin{array}{c}\text { HNF- } \\
\text { ACA }\end{array}$ & $\begin{array}{c}\text { D- } \\
\text { FICCA }\end{array}$ & $\begin{array}{c}\text { G- } \\
\text { FQL }\end{array}$ & TRAACK \\
\hline 20 & 0.6 & 0.55 & 0.52 & 0.4 \\
\hline 40 & 0.8 & 0.7 & 0.6 & 0.5 \\
\hline 60 & 0.9 & 0.8 & 0.7 & 0.6 \\
\hline 80 & 1 & 0.9 & 0.8 & 0.7 \\
\hline 100 & 1.21 & 1 & 0.9 & 0.8 \\
\hline
\end{tabular}

\section{CONCLUSION AND FUTURE WORK}

In this work, HNF-ACA based anomaly detection scheme is proposed for large scale sensor networks. The proposed filter hybridizes the Mamdani and Sugeno fuzzy interference model which offers the effective filtering mechanism. Furthermore, the proposed mechanism improves the performance of Sugeno model as well as Mamdani model. The ACA technique greatly improves the membership values to the input. It exploits the stability in their neighborhood information. Initially, the cluster based routing is selected with the trust vector and residual energy of neighbor nodes in the random topology. In this system, the packet transmission from a source node to a destination node is encrypted using the RSA scheme. After that, the identification of malicious nodes is made by using trust recommendation value. Finally, the normal and malicious nodes are classified by using HNFACA. An experimental performance result proved that the HNF-ACA provides maximum detection efficiency and high network lifetime, an end to end 
delay and overhead is minimized than the existing D-FICCA, G-FQL and TRAACK.

\author{
ETHICS APPROVAL AND CONSENT TO \\ PARTICIPATE \\ Not applicable.
}

HUMAN AND ANIMAL RIGHTS

No animals/humans were used for studies that are basis of this research.

\section{CONSENT FOR PUBLICATION \\ Not applicable.}

AVAILABILITY OF DATA AND MATERIALS

The authors confirm that the data supporting the findings of this research are available within the article.

\section{FUNDING}

None.

\section{CONFLICT OF INTEREST}

The authors declare no conflict of interest, financial or otherwise.

\section{ACKNOWLEDGEMENTS}

The authors would like to thank their present employer for providing support while carrying out this research work.

\section{REFERENCES}

[1]. McAfee, Centre for Strategic \& International Studies, Estimating the Global Cost of Cybercrime, Technical Report, McAfee, Centre for Strategic \& International Studies, 2014.

[2]. Patel A, Qassim Q, Wills C. A survey of intrusion detection and prevention systems. Inf. Manage. Comput. Secur. 2010; 18(4): $277-290$

[3]. Wang S S, Yan K Q, Wang S C, Liu C W. An integrated intrusion detection system for cluster-based wireless sensor networks. Expert Syst. Appl. 2011; 38(12): 15234-15243.

[4]. Salmon H M, De Farias C M, Loureiro P, Pirmez L, Rossetto S, Rodrigues P H D A, Pirmez R, Delicato F C, da Costa Carmo L F R. 2013. Intrusion detection system for wireless sensor networks using danger theory immune-inspired techniques. Int. J. Wireless Inf. Networks. 2013; 20(1): 39-66.

[5]. Shamshirband S, Amini A, Anuar N B, Kiah M L, Teh Y W, Furnell S. D-FICCA: A density-based fuzzy imperialist competitive clustering algorithm for intrusion detection in wireless sensor networks. Measurement. 2014; 55: 212-226.

[6]. Riecker M, Biedermann S, El Bansarkhani R, Hollick M. Lightweight energy consumption-based intrusion detection system for wireless sensor networks. Int. J. Inf. Secur. 2015; 14(2): $155-167$.

[7]. Shamshirband S, Patel A, Anuar NB, Kiah ML, Abraham A. Cooperative game theoretic approach using fuzzy Q-learning for detecting and preventing intrusions in wireless sensor networks. Eng. Appl. Artif. Intell. 2014; 32: 228-241.
[8]. Assad N, Elbhiri B, Faqihi MA, Ouadou M, Aboutajdine D. Efficient deployment quality analysis for intrusion detection in wireless sensor networks. Wireless Networks. 2016; 22(3): 9911006.

[9]. Rajeshkumar G, Valluvan K R. An energy aware trust based intrusion detection system with adaptive acknowledgement for wireless sensor network. Wireless Pers. Commun. 2017; 94(4): 1993-2007.

[10]. Khanum S, Usman M, Hussain K, Zafar R, Sher M. Energyefficient intrusion detection system for wireless sensor network based on MUSK architecture. InHigh Performance Computing and Applications 2010 (pp. 212-217). Springer, Berlin, Heidelberg.

[11]. Panda M. Security in wireless sensor networks using cryptographic techniques. American J. Eng. Res. 2014; 3(01): 50-56.

[12]. Singh G. A study of encryption algorithms (RSA, DES, 3DES and AES) for information security. Int. J. Comput. Appl. 2013; 67: $33-38$.

[13]. Kumar PA, Selvakumar S. Detection of distributed denial of service attacks using an ensemble of adaptive and hybrid neurofuzzy systems. Comput. Commun. 2013; 36(3): 303-19.

[14]. Saidi-Mehrabad M, Dehnavi-Arani S, Evazabadian F, Mahmoodian V. An Ant Colony Algorithm (ACA) for solving the new integrated model of job shop scheduling and conflict-free routing of AGVs. Comput. Industrial Eng. 2015; 86: 2-13.

[15]. Nirmaladevi P, Tamilarasi A. Secure Intrusion Detection System for Authentication in Wireless Sensor Networks. Asian J. Res. Social Sci. Humanities. 2016; 6(9): 131-146. 